Check for updates

Cite this: RSC Adv., 2017, 7, 46604

Received 5th September 2017

Accepted 27th September 2017

DOI: $10.1039 / \mathrm{c} 7 \mathrm{ra0} 8889 \mathrm{~h}$

rsc.li/rsc-advances

\title{
Understanding the spin-dependent electronic properties of symmetrically far-edge doped zigzag graphene nanoribbon from a first principles study $\dagger$
}

\author{
Amrit Sarmah (iD *a and Pavel Hobza ${ }^{\text {ab }}$
}

\begin{abstract}
We have systematically elucidated the electronic and spin dependent behavior of far-edge doped zigzag graphene nanoribbons from a DFT based first principles study. The relative changes in the electronic environment due to an increment in the impurity concentration as well as the size of the hexagonal carbon network have been thoroughly assayed. Although the substitutional doping is most favorable at the edges of the nanoribbons, our DFT simulations envision that far-edge doping also induced some tunable spin-dependent properties in the zigzag graphene nanoribbons. The total magnetic moment of the systems seems to have some sequential dependencies on the doping concentration along with the relative growth in the crystal lattice. It is observed that the impurity (boron or nitrogen) doping at the faredge sites can break the electronic degeneracy in the up and down spin channels. Interestingly, in both cases spin-up electrons are found to be metallic or semi-metallic and the spin-down electrons demonstrate an insulating nature.
\end{abstract}

\section{Introduction}

The massless Dirac fermion ${ }^{\mathbf{1 , 2}}$ nature of 2D graphene nanostructures has recently unlocked some exciting avenues in the field of nanomaterial research. The 2D layered graphene structure exhibits some unprecedented electronic properties such as long spin relaxation time, high conductivity, and quantum Hall effect and makes it an ideal candidate for future nanoelectronics and device applications. ${ }^{3-6}$ In a speculative situation, one-dimensional graphene nanoribbons (GNRs) can be fabricated by cutting through an infinite graphene sheet where the dangling $\sigma$ bonds (at the edges) are passivated with atomic hydrogen (known as hydrogenated or hydrogenterminated edges) and keeping all the carbon atoms in an $\mathrm{sp}^{2}$ hybridized state. Depending on the shape of the edges and the extended length, GNRs behave differently in both electronic and magnetic aspects.

The zigzag GNR embarks considerable excitement among scientific community due to the presence of edge states that lead to spin polarization and local magnetization in case of ZGNR. ${ }^{7}$ By applying some external perturbations like electric field, magnetic field or chemical modifications such as doping,

${ }^{a}$ Institute of Organic Chemistry and Biochemistry of the Czech Academy of Sciences, Flemingovo nam. 2, CZ-16610 Prague 6, Czech Republic. E-mail: amrit.sarmah@ marge.uochb.cas.cz

${ }^{b}$ Department of Physical Chemistry, Palacký University, CZ-77146 Olomouc, Czech Republic

† Electronic supplementary information (ESI) available. See DOI: 10.1039/c7ra09889h etc. imparted modulations to the local magnetization in ZGNR. It is important to note that in some recent findings predict that the physical properties of edges decorated ZGNR can be tuned for efficient spin filtering ${ }^{\mathbf{8}, 9}$ negative differential resistance ${ }^{\mathbf{1 0}}$ and many other promising aspects for future spintronics.

An ample amount of high quality experimental and theoretical studies have been performed to understand the electronic environment and the possible tailoring of electronic properties of edges decorated ZGNR for the nano-optics and electronics. ${ }^{11-15}$ In the last couple of years, there is a sequential growth in the research activities to optimize the capability of ZGNR as an energy efficient material for device fabrication. ${ }^{\mathbf{1 6 - 2 0}}$ A group of researchers explored possibilities of fine tune the electronic properties of ZGNR by incorporating ions or molecules at the edges. ${ }^{21}$ Another broadly employed approach for the edge modification in ZGNR is to make a structural hybrid combining zigzag and armchair nanoribbon (as the initial structural motif) and then cutting it in different shapes according to the structural orientations. ${ }^{22-25}$ Particularly in ZGNR, relatively large contributions from the edge states account the high density of states (DOS) at the Fermi level. ${ }^{26}$ Now, some spin unresolved calculations suggest magnetic instability to the system in turns splitting the edge states around the Fermi level. ${ }^{27}$ This holds the spin polarity in ZGNR with finite magnetic moments at the edge carbon atoms and a ferromagnetic spin alignment along the border. ${ }^{28}$

Doping technique is also an essential tool for the ZGNR based device fabrication. Perhaps, there are some inherent benefits of the edge doping in ZGNR. ${ }^{29}$ It is well accepted that doping at the zigzag edges are more stable and the doping impurities 


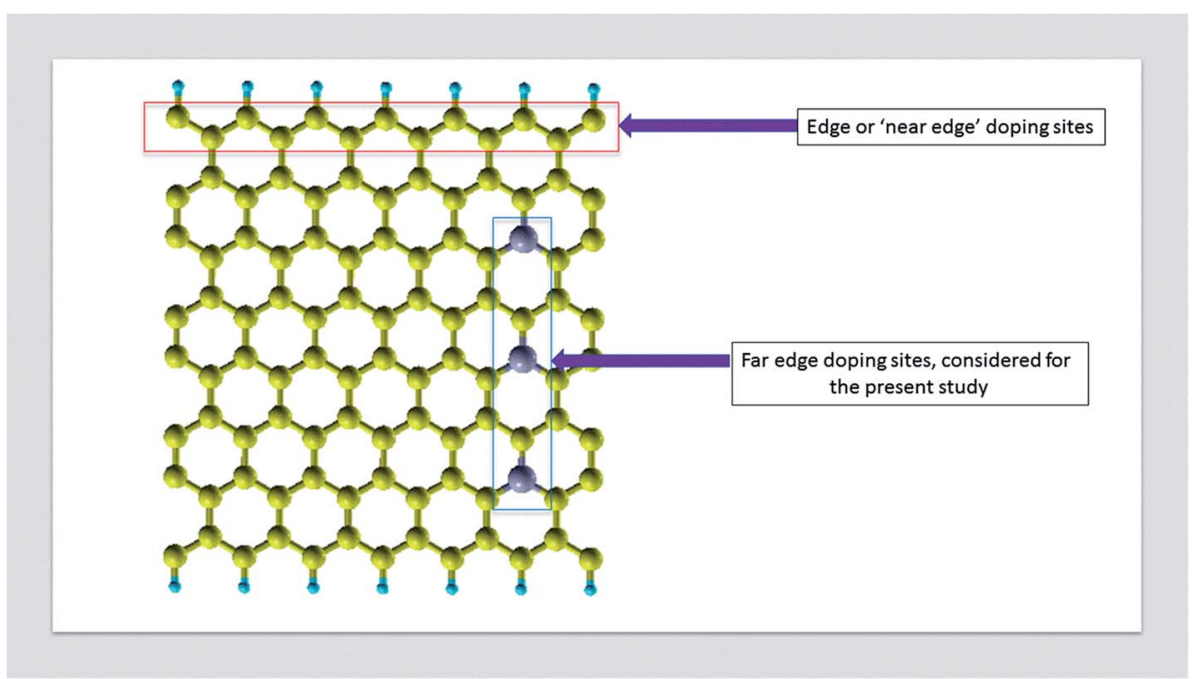

Fig. 1 A simple pictorial representation of the model doped nanoribbon system considered for our study. Here, the possible edge or 'near edge' and 'far edge' doping sites are highlighted with red and green rectangles, respectively.

significantly alter the spin polarization at the edges. ${ }^{30,31}$ In a recent report, Zou et $a l^{32}$ studied the spin filtering effect and negative differential behavior of phosphorus-doped ZGNR. In an another elaborate study, Silva $e t a .^{33}$ explored the structural, magnetic and corresponding transport behavior of the doped armchair and zigzag GNR. Using conventional and hybrid (exactexchange) DFT functionals, Jiang et al. ${ }^{34}$ investigated the effect of nitrogen doping on the edges and the corresponding changes in the characteristic electronic behavior of edge-decorated GNR. They have studied different systems namely chiral, armchair and zigzag GNRs and tested the effect of N-doping at different sites. The relative changes in the formation energies, as well as the electrical properties due to the nitrogen doping at different sites in a GNR system, has also been reported by Yu et al. ${ }^{31}$ Interestingly, their study exhibits an important correlation between the distribution of nitrogen nonbonding electrons with the position of nitrogen doping.

It is perceptible from the above discussions that the electronic and magnetic properties of ZGNR can be modulated by incorporating external impurities in different lattice sites of a GNR unit cell. ${ }^{35}$ There are specific advantages by carrying out doping on 1D GNR surface using nitrogen and boron atoms. The boron and nitrogen possess almost similar atomic radii to that of carbon and containing one electron extra (for N) or less (for B) than carbon. In principle, when GNR is doped with nitrogen or boron atoms, introduce specific impurity level at the edges states (either near the top of valence band or close to the bottom of the conduction band). It is important to note that the edge decorated B-doped GNR shown to have phase transition from metallic to semiconductor states associated with the breaking of symmetry in spin polarized transmittance channels. ${ }^{36}$ Again, other electrical and magnetic properties of the ZGNR systems depend on the particular doping site near the edges. ${ }^{37}$ However, there are limited reports on the systematic rationalization of the simultaneous structural growth and doping concentration on the electronic and magnetic properties of ZGNR. To the best of author's knowledge, our present study will be able to address some important aspects of symmetric doping effect on the sites diagonally opposite to the edge carbon atoms in zigzag graphene nanoribbons. It is equally important to investigate the impact of simultaneous variations in the GNR width and doping concentrations to the overall electronic environment of the system. In the proceeding sections, we are going to elaborate our findings to address some the abovementioned points. Essentially, these findings will put some new insights to the improvement and modulation of the electronic properties in B and N doped ZGNR.

\section{Computational details}

$A b$ initio calculations were performed using the open source Quantum Espresso 5.4.0 code $^{38}$ We have used projector augmented-wave pseudopotentials and exchange-correlation (xc) effects were incorporated at the Perdew-Burke-Ernzerhof $(\mathrm{PBE})^{39}$ level. The van der Waals correction to the calculations has been taken care through semi-empirical Grimme's DFT-D2 methodology ${ }^{40}$ as implemented in Quantum Espresso. The kinetic energy cutoff for wavefunctions is set at 40 Ry and the corresponding cutoff for charge density and potential was set at 160 Ry. We took $n$-ZGNR (where $n=8,12$ and 16) systems for this study and the particular number denotes the number of carbon atoms along the zigzag edges. The integration over the Brillouin zone was set an optimized Monkhorst Pack ${ }^{41}$ of $1 \times 1$ $\times 10 k$-points (GNR growth direction $z$-direction along). The forces were minimized up to $<10-3$ Ry per a.u. for all atoms to obtain fully relaxed atomic geometries. Computed formation energy $E_{\mathrm{f}}$ for the doping state is based on the following equation

$$
E_{\mathrm{f}}=\left(E_{\text {(doped ZGNR) }}+E_{(\text {carbon })}\right)-\left(E_{(\mathrm{ZGNR})}+E_{(\text {dopant })}\right)
$$

where, $E_{(\mathrm{ZGNR})}$ is the total energy of perfect ZGNR, $E_{(\mathrm{doped} \mathrm{ZGNR)}}$ is the energy of doped ZGNR, $E_{\text {(carbon) }}$ and $E_{\text {(dopant) }}$ is the energy of 
the single carbon and dopant atom, respectively. In all the structures, the dangling bonds at the edge atoms are saturated with hydrogens. In this particular study, our simulation supercell contains 60, 80 and 108 atoms for 8, 12 and 16 ZGNR, respectively (Fig. S1 in ESI $\dagger$ ). We have adopted one-dimensional periodic supercells (growth in the $z$-direction) with $10 \AA$ vacuum regions in both $x$ and $y$ directions to limit the self-interaction between the repetitive units. A simplified pictorial representation of the model system describing the specific doping position is given in Fig. 1. The pictorial representations of relaxed structures for the three different ZGNR systems are reported in ESI section (Fig. S1†).

\section{Results and discussions}

\section{(a) Undoped ZGNR}

In this section, we will revisit the electronic and magnetic properties of undoped ZGNR. Zigzag edge GNRs are the subject of extensive research in the field of one dimensional (1D) carbon nanostructures. These discussions are basically important to calibrate our current findings and to extend the logical analogies further to interpret the relative changes in the boron and nitrogen doped nanoribbons. We have computed relative formation energies for the different ZGNR systems. The graphical representations of the relative formation energies (per single atom doping) for both boron and nitrogen doped systems at different sites are depicted in Fig. 2.

It is observed that the formation energy for the $\mathrm{N}$-doped system is almost two times higher than the boron doped one. However, we did not observe any substantial changes in the formation energy pattern either due to the increase in ZGNR width or the number of doping impurities. In N-doped ZGNR the computed formation energy values are consistent with the findings of $\mathrm{Yu}$ et $a l^{31}$ The lowest energy configuration is appeared to be the near-edge single nitrogen doped 8-ZGNR. Whereas, the 12 ZGNR containing two boron atoms in the cell exhibits lowest formation energy compared to other two systems of B-doped graphene nanoribbon. It is worth mentioning here that the qualitative features of the formation energy trend are correlated well with the earlier reports. ${ }^{33,34}$ The preferential doping sites for the GNRs are at the edges. ${ }^{28-30}$ It is worth mentioning here that, the introduction of (boron or nitrogen) impurities at a particular location away from the edges of the nanoribbon are found to be stable and showing some far-reaching impact on the electronic and magnetic properties of the ZGNR.

As a basic starting point, we performed spin-resolved DFT calculations on the three undoped ZGNR systems to understand the electronic and magnetic properties of the system. The undoped ZGNR systems have been extensively studied in the last couple of years. ${ }^{35}$ These starting simulations can be considered as a reference point to specify the relative changes observed to the electronic environment of nanoribbons, before and after the incorporation of doping impurities in the framework. The calculations are performed on all three undoped ZGNR systems (i.e., 8, 12, and 16). As the different computed parameters of the three systems did not exhibit any significant deviation from each other, we will proceed the discussion by taking the example of 12-ZGNR. The findings of other two systems are included in the ESI section. $\dagger$

The spin-polarized electronic density of states (DOS) plot for the undoped 12-ZGNR system is given in Fig. 3(a). The metallic nature of the ZGNR can be understood from the DOS spectrum. The spin-resolved DOS exhibits two highly intense peaks just below and above the Fermi level. Indeed, these sharp peaks are accounted primarily due to the edges states pi-electron

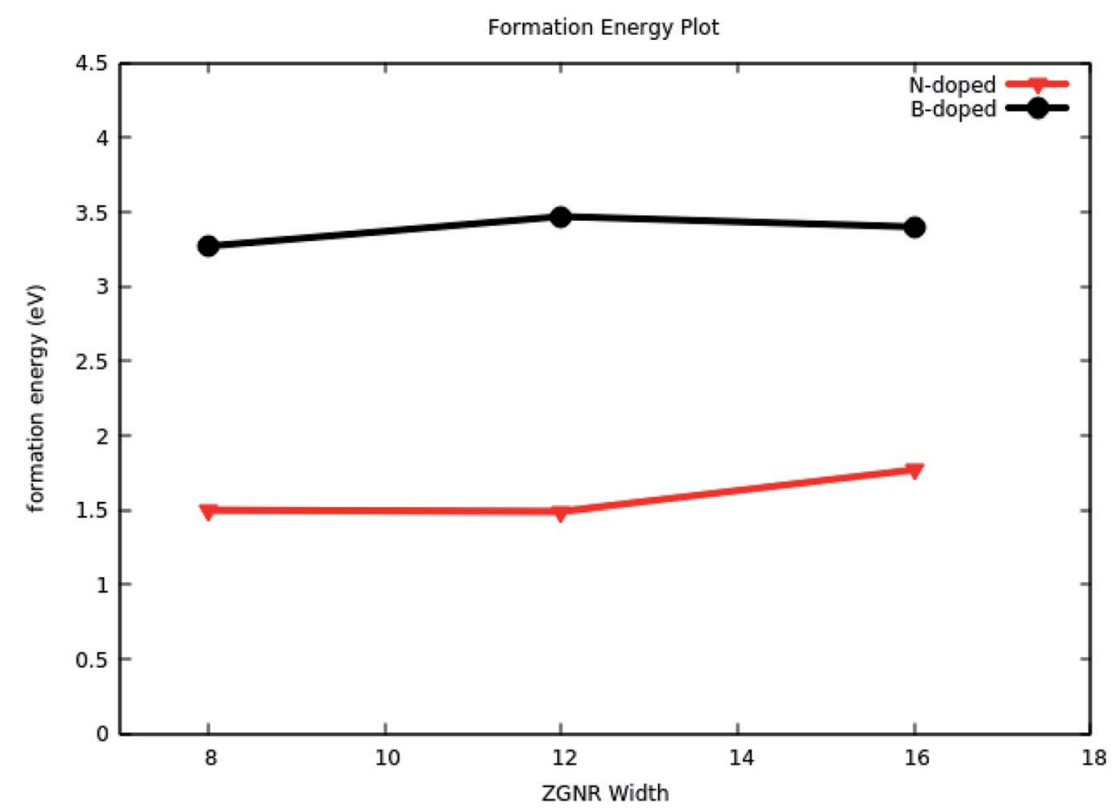

Fig. 2 Pictorial representation of the far-edge doping sites in the three different ZGNR systems and corresponding relative formation energies for $\mathrm{N}$-doped (red) and B-doped (black) against ribbon width. 


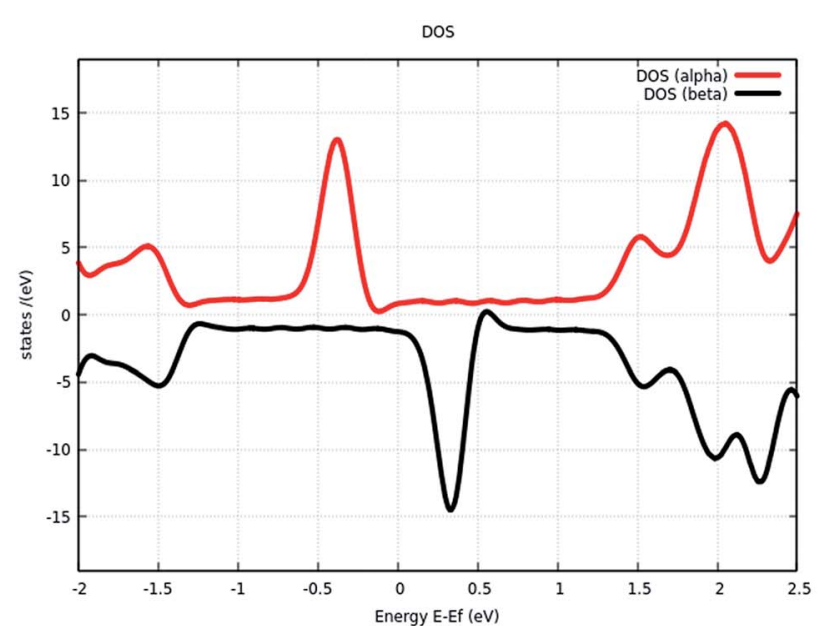

(a)

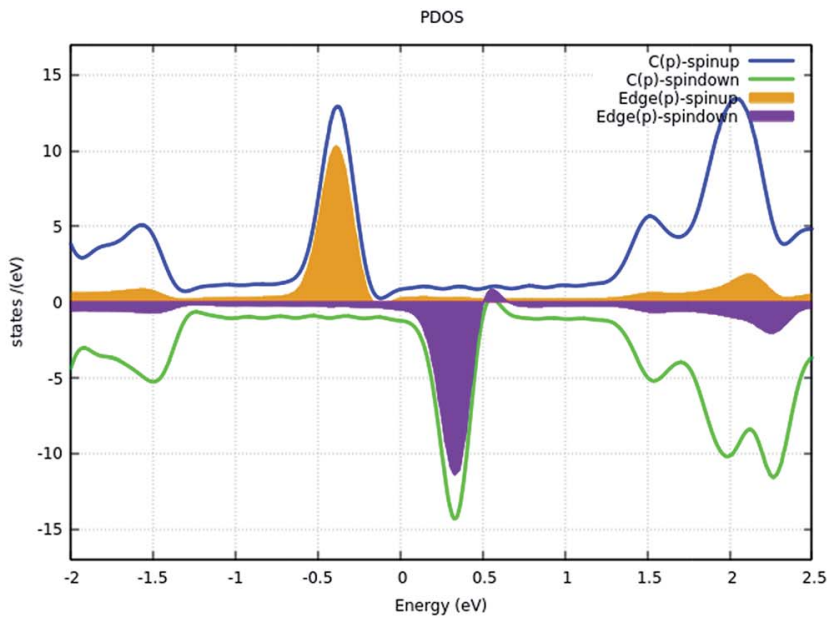

(b)

Fig. 3 Spin-resolved (a) density of states (DOS) (b) partial density of states (PDOS) for 12-ZGNR system. In the left panel (DOS) the up-spin (down-spin) electrons are represented by red (black) lines. In the right panel (PDOS) carbon atoms p electrons contributions are represented by blue and green lines and the contributions from the edges atoms are shown by the filled regions.

contributions. This particular phenomenon is the consequence of quantum confinement effects and reportedly sensitive to ZGNR width. ${ }^{42}$ Fig. $3(\mathrm{~b})$ represents the spin polarized partial density of states (PDOS) plot for 12-ZGNR. Here, blue and green lines depict the density of spin up and down p-electrons of carbon and the filled regions inside plots are the contribution from p-electrons (spin up and down) of edges carbon atoms. This clearly indicates that the edges state p-electrons are the almost solo contributors to the DOS spectrum of ZGNR ${ }^{43,44}$ at just above and below the Fermi level. The DOS and PDOS plots of other two ZGNR systems (8 and 12) are included in ESI section (Fig. S2 $\uparrow$ ). The visual representation of the local density of states (LDOS) for the three undoped ZGNR systems is given in Fig. 4. Particularly, the change in LDOS pattern with the variation of GNR width can be understood from LDOS plots. Here, we can observe that on going from 8 to 16 ZGNR, there is some clear shift in the LDOS location. As the width of GNR increased the DOS states are moving more and more towards the edges, gradually decreasing at the central region of the nanoribbon. Subsequently, we have reported the isosurface density plot for the two wave functions just below and above the Fermi energy in Fig. 5. Here, we observed some symmetric patterns in the
HOMO and LUMO states for the 8-ZGNR. However, for 12 and 16 ZGNR, the occupied states are located in the central part of nanoribbon and unoccupied states are localized at the ZGNR edges.

Our DFT calculations predicted a high degree of total magnetic moments for the undoped ZGNR systems. The computed magnetic moment values are 2.54, 2.98 and 3.23 Bohr magneton per cell for 8, 12 and 16-ZGNR, respectively. Interestingly, there is an almost linear increase in the magnetic moment values with the increase in ZGNR width. Fig. 6 depicts the spin density distribution plots for the three undoped ZGNR systems. To be precise, the spin density appeared to concentrate at the two edges of the three different systems of ZGNR. It is worth mentioning here that the energetically favorable antiferromagnetic configuration between two highly ordered ferromagnetically aligned localized edges states (more details in Fig. 6) in ZGNR is consistent with the earlier reports. ${ }^{45}$ Due to the same spin alignment there supposed to have some strong ferromagnetic interactions between the edges that in turns induce a large magnetic moment on the system. Perhaps, the splitting of pi-electrons into spin-up and spin-down channels near the Fermi level impart strong spin-polarity to the ZGNR. ${ }^{46}$
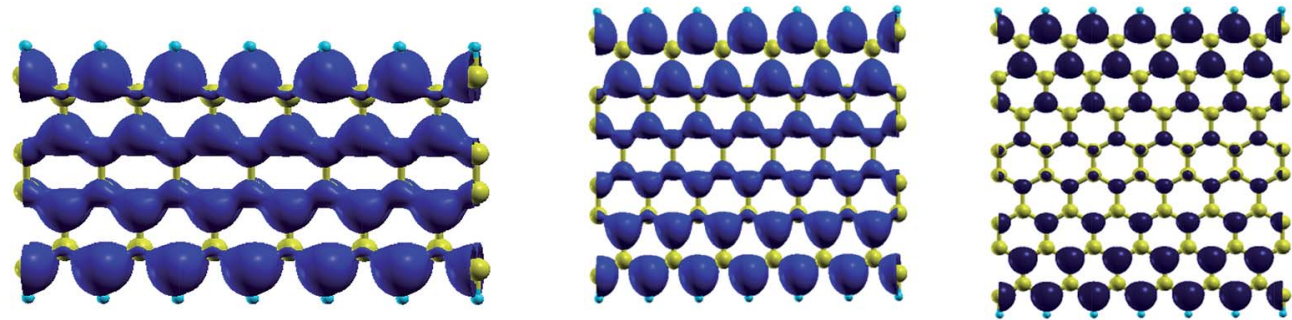

Fig. 4 The visual graphics of local density of states (LDOS) distribution in the supercell for 8,12 and 16-ZGNR systems (left to right). The isodensity value is set on 0.003 e Bohr $^{-3}$. 

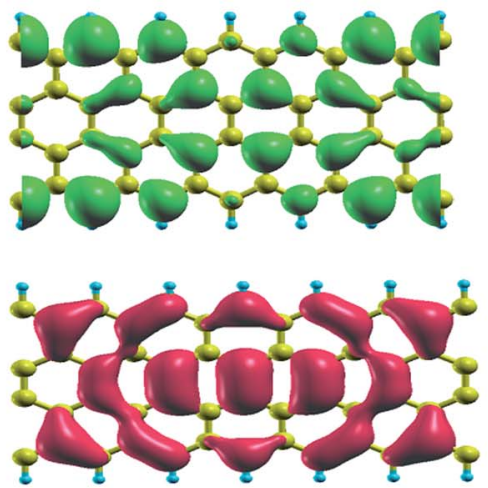

8-ZGNR
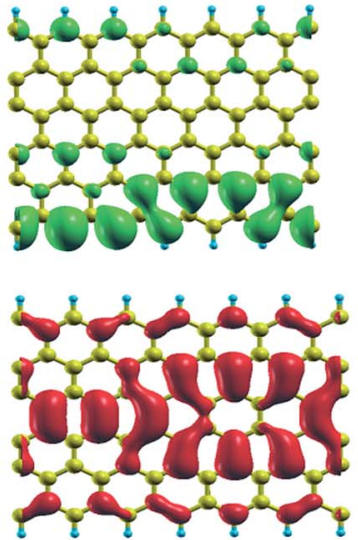

12-ZGNR
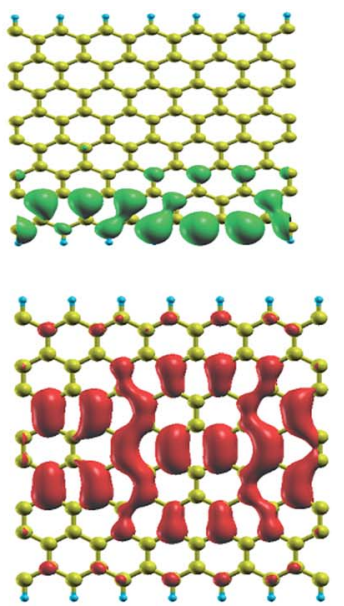

16-ZGNR

Fig. 5 The highest occupied level (VBM) and corresponding lowest unoccupied level (CBM) colored in red (lower panel) and green (above panel), respectively for the three systems.

This interesting magnetoelectric property of the ZGNR system can be exploited to design and fabricate potential spin filtering devices in near future. ${ }^{32}$

\section{(b) Boron-doped ZGNR}

We have performed some symmetric doping in the ZGNR and systematically replaced one, two and three carbon atoms from 8, 12 and 16 ZGNR systems, respectively with boron atoms. The boron doing injects a hole to the system. The relaxed structures with symmetric doping sites can be found in ESI section (Fig. S1†). As we have mentioned previously, the doped boron impurities are placed diagonally opposite to the edge carbon atoms in the ZGNR network. The motivation behind our present study is to explore the importance of impurity doping on carbon network of ZGNR at a position located away from the edges. There is a large number of reports on the edge doped ZGNR, but we believed that the doping at near-edge sites (away from edges) can also lead us to some important findings regarding the electronic behavior of doped ZGNR.

Initially, a single carbon atom in the hexagonal nanoribbon framework of 8-ZGNR has been substituted with boron atom.
Since the 8-ZGNR supercell contains altogether 48 carbon atoms, single boron doping introduces approximately $2 \%$ impurity concentration in the system. Similarly, we have doped 2 and 3 boron atoms in the supercell of 12 and 16-ZGNR (containing 72 and 96 carbon atoms) which roughly corresponds to $2.78 \%$ and $3.2 \%$ doping concentrations, respectively. Here, according to our simulation model the increasing size of ZGNR supercell simultaneously accompanied by a gradual increase in the corresponding doping concentrations. This provides us the scope to understand the effect of simultaneous growth in cell size and doping concentration on the electronic and magnetic properties of boron doped ZGNR.

We have reported the formation energy plot for the three different B-doped ZGNR systems in Fig. 2. It seems that an increase in the doping concentration does not have a substantial impact on the formation energy values. We observed very little changes in the formation energy values on going from 8 to 12-ZGNR system. However, this particular observation slightly deviates from the earlier reports, ${ }^{31}$ where there is some linear increase in the formation energy with the increasing ribbon width. It is understandable that in our study the number of

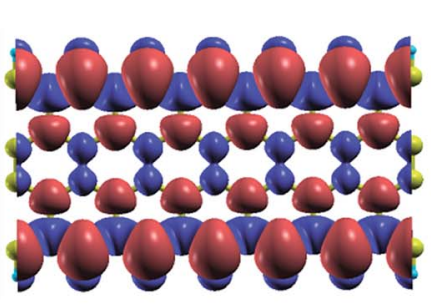

8-ZGNR

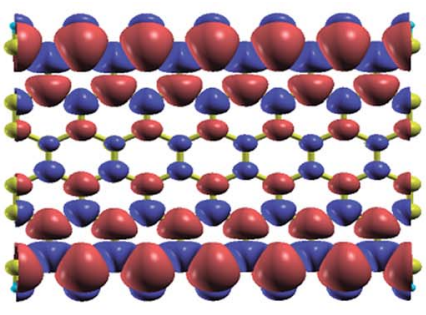

12-ZGNR

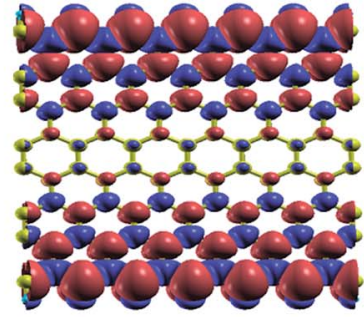

16-ZGNR

Fig. 6 The spin density distribution plots for the three different ZGNRs. The red and purple color regions in the plot represents the oppositely aligned excess spin densities. 

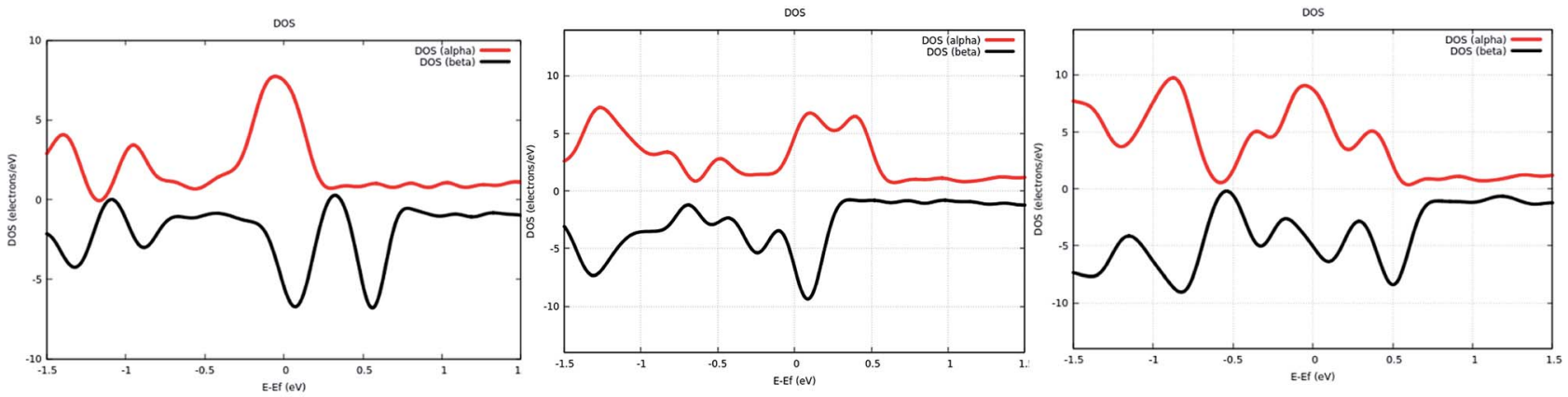

PDOS

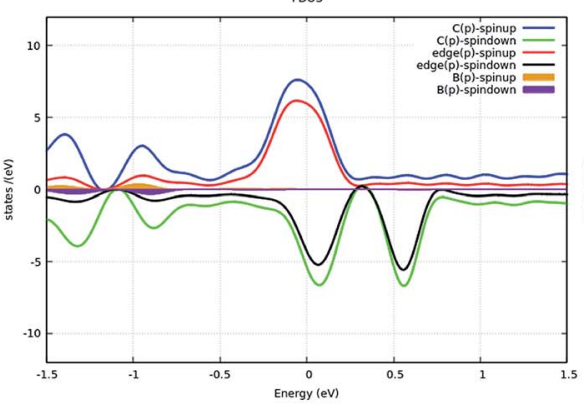

8-BZGNR

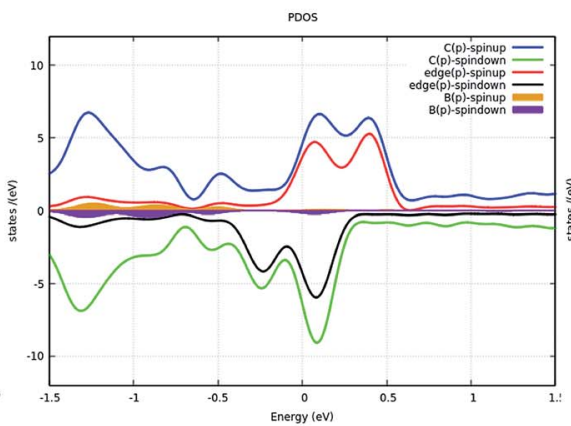

12-BZGNR

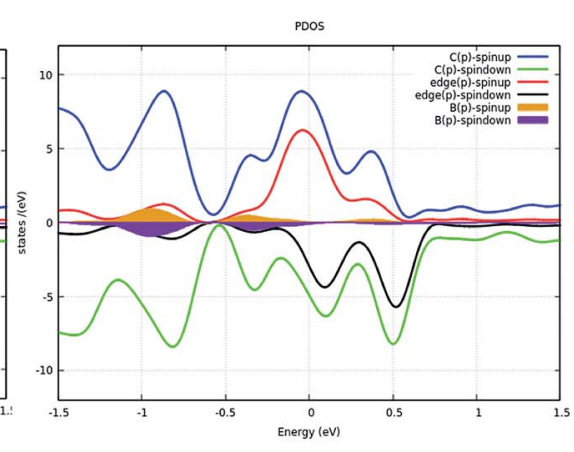

16-BZGNR

Fig. 7 The electronic structures of three boron doped systems. Upper panel represents the spin polarized total density of states (DOS) and below panel is the corresponding partial density of states (PDOS). In the upper panel red and blue lines represents the spin up and down electron configurations. For PDOS blue (spin-up) and green (spin-down) lines represent the p-electrons contribution from carbon atoms. The red (spinup) and black (spin-down) are the electrons contribution from the particular carbon atoms at the edges. The filled regions showing the contribution from the doped boron impurities.

doping sites is also increasing with the increase in ribbon width. So, at some point, it might possible that the energy perturbation induced in the supercell (due to foreign atom substitution) is compensated up to some extent by the increasing number of doping sites (defects).

The spin-resolved electronic density of states (DOS) plots for the three different B-doped systems are reported in Fig. 7. The striking feature of 8-ZGNR is the appearance of sharply split peaks near the Fermi level. In principle, the substitution of an impurity in the hexagonal carbon network induced defects to the lattice and this potentially act as a scattering center for the propagating electronic wave functions in a particular direction. ${ }^{47}$ There is some strong spin-polarity in B-doped 8-ZGNR due to the occurrence of sharp peaks near the Fermi level and results from the definite magnetic moment (1.28 BM per cell) in the system. The DOS splitting patterns are changing to more symmetric one upon increasing the doping concentrations as well as the width of ZGNR. This is associated with some decrease in the total magnetic moment values 1.10 and $1.08 \mathrm{BM}$ per cell for 12 and 16 B-doped systems, respectively. However, the characteristic down-spin electron peak (almost bisected by the Fermi level) remains un-shifted for the three systems. The

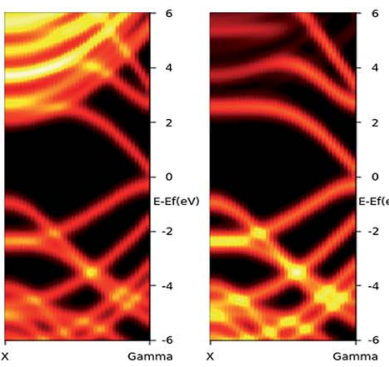

8-BZGNR
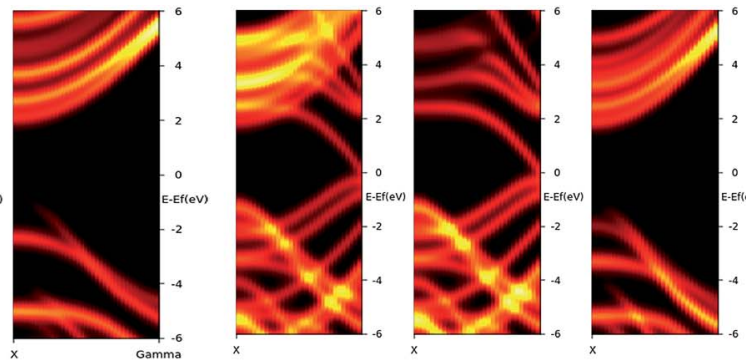

12-BZGNR
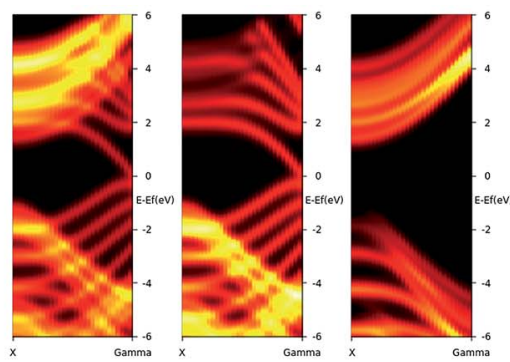

16-BZGNR

Fig. 8 Computed spin polarized electronic band structures for the three different B-doped ZGNR systems. Here, the three unites for a particular system individually represents the overall, spin-up and spin-down electrons distributions, respectively. The Fermi level is set at zero. 


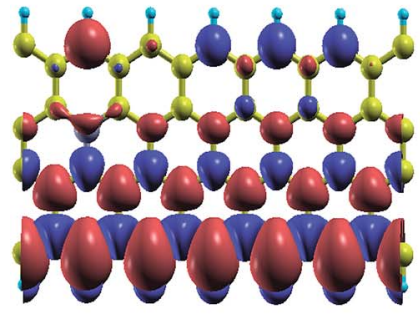

8-BZGNR

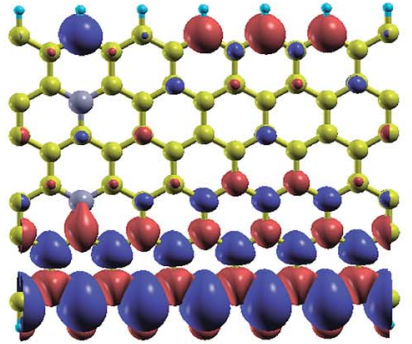

12-BZGNR

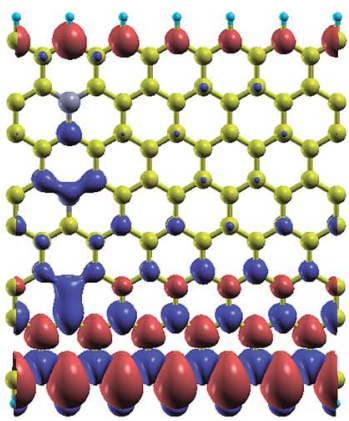

16-BZGNR

Fig. 9 The spin density distribution plots for the three different B-doped ZGNRs. The red and purple color regions in the plot represents the oppositely aligned excess spin densities.

effect of edge states pi electrons are prevalent in all three cases of boron doping, as appeared from the PDOS plots (in Fig. 7). We have observed some minor contributions from the doped Batoms in case of 12 and 16 ZGNR systems near the valence band. This shows some good agreement with the recent findings of Cloke et $a l .{ }^{48}$

Computed spin-resolved band structures of relatively smaller B-doped systems are represented in Fig. 8. Here, the symmetric doping sites remain constant but we have taken a reduced supercell with a highly dense grid. The interesting aspect of the band structure is the 'half-metallic' nature of the doped nanoribbon system. In case of ZGNR, this peculiar behavior has been recently reported by Son et $a l^{49}$ It appears from the spinresolved band structure that the spin-up electrons are in metallic (or semi-metallic) in nature and down-spin electrons exhibit insulator properties. The homogeneous electric field induced half-metallic nature of doped zigzag GNR along the edges is well documented in the literature. ${ }^{49}$ Perhaps, the symmetric doping at the diagonally opposite edge atoms can also able to induce some perturbations to the local electric and magnetic fields of the ZGNR and triggers such interesting behavior in our model ZGNR systems. In our upcoming study, we will put some elaborate insight to this particular observation.

Boron doping imparts some specific changes in the spinpolarity of the system (Fig. 9). It is observed that the characteristic ferromagnetic-anti-ferromagnetic interaction in one of the edges being suppressed by the development of some
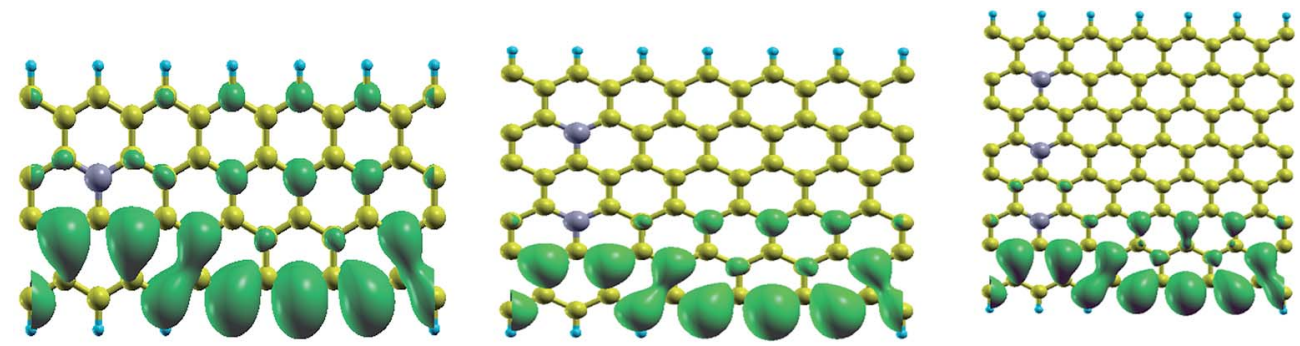

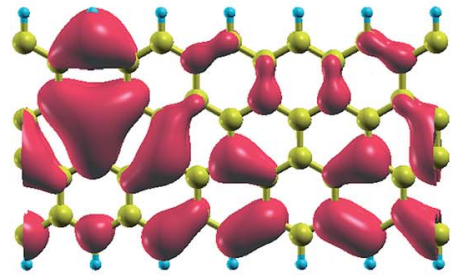

8-BZGNR

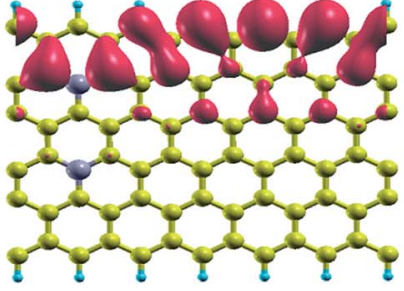

12-BZGNR

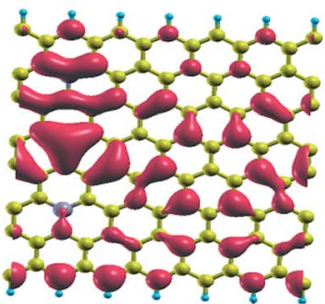

16-BZGNR

Fig. 10 The highest occupied level (VBM) and corresponding lowest unoccupied level (CBM) colored in red (lower panel) and green (above panel), respectively for the three boron doped ZGNR systems. 

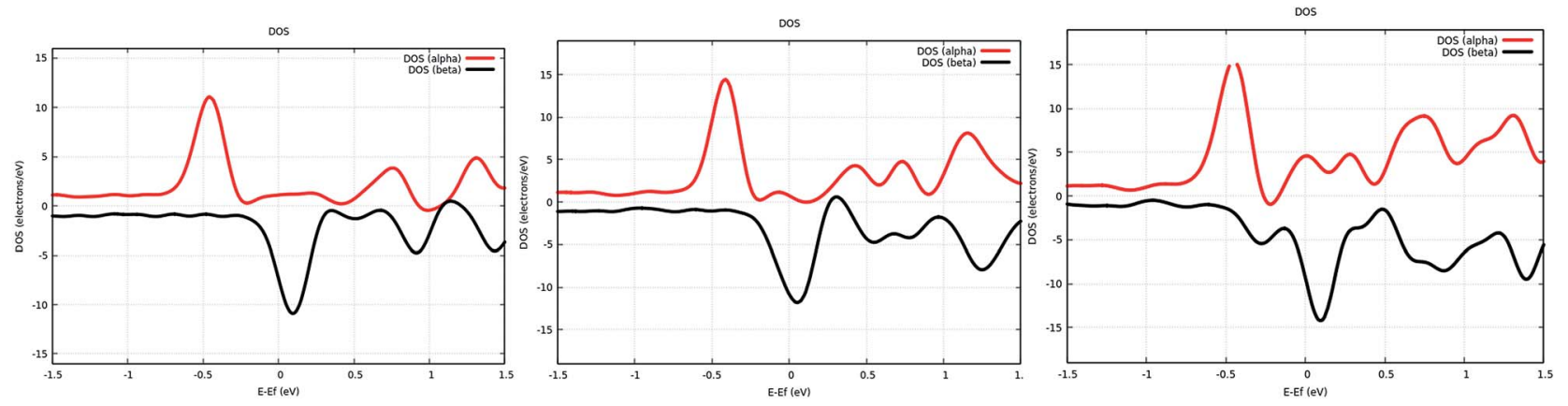

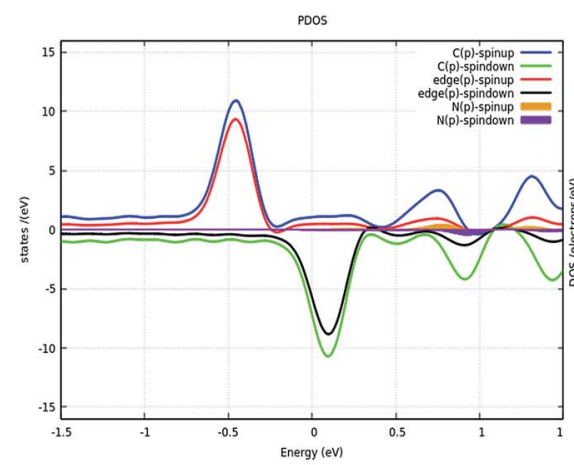

8-NZGNR

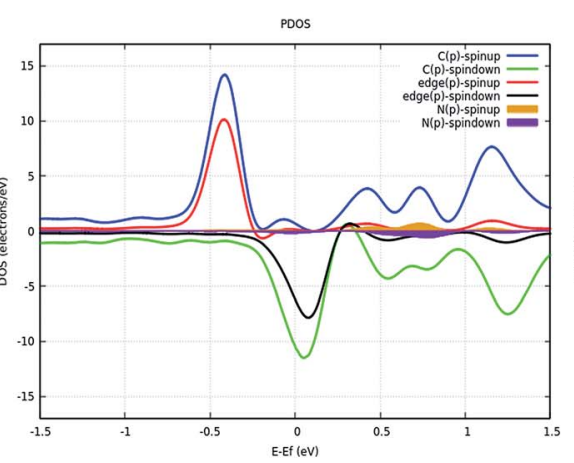

12-NZGNR

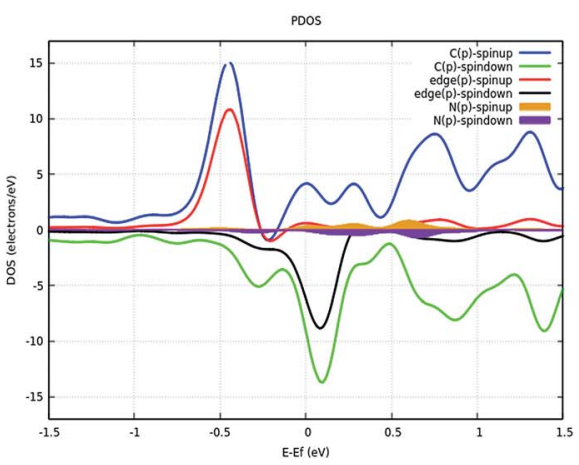

16-NZGNR

Fig. 11 The electronic structures of three nitrogen doped systems. Upper panel represents the spin polarized total density of states (DOS) and below panel is the corresponding partial density of states (PDOS). In the upper panel red and blue lines represents the spin up and down electron configurations. For PDOS blue (spin-up) and green (spin-down) lines represent the p-electrons contribution from carbon atoms. The red (spinup) and black (spin-down) are the electrons contribution from the particular carbon atoms at the edges. The filled regions exhibit contributions from the doped nitrogen impurities.

ferromagnetic states. It is worth mentioning here that the increase in the doping concentration does not seem to have any significant impacts on the electron spin arrangements at the edges. However, some careful observation recognized minor growth in spin densities (characteristically opposite to that of the edges) over the doping locations upon an increase in the doping concentration as well as the GNR width. Subsequently, the standard spin-polarity equilibrium exists between the opposite edges (as observed in case of undoped systems) is strongly disturbed due to the presence of boron impurities in GNR network. The propagations of a wavefunction at the valence band maxima (VBM) and conduction band minima (CBM) are reported in Fig. 10. Here, the sharp edge localized states are prevalent in CBM region while there are some significant contributions from the boron doped sites in VBM states.

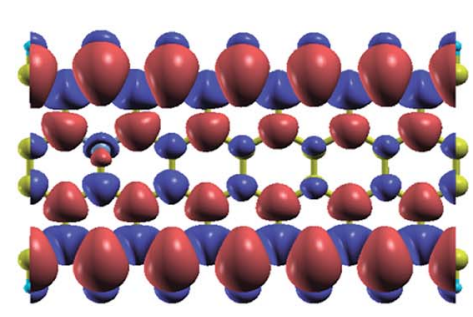

8-NZGNR

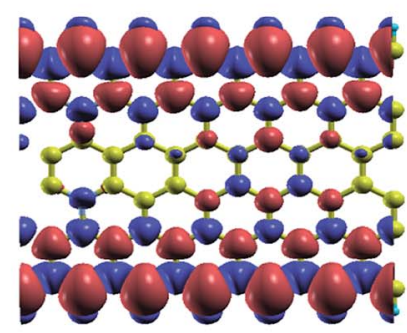

12-NZGNR

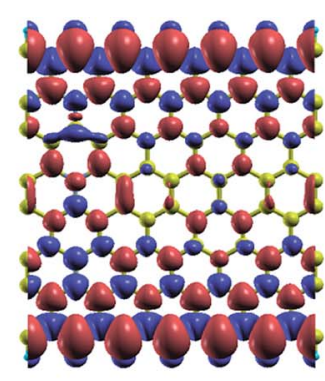

16-NZGNR

Fig. 12 The spin density distribution plots for the three different N-doped ZGNRs. The red and purple color regions in the plot represents the oppositely aligned excess spin densities. 

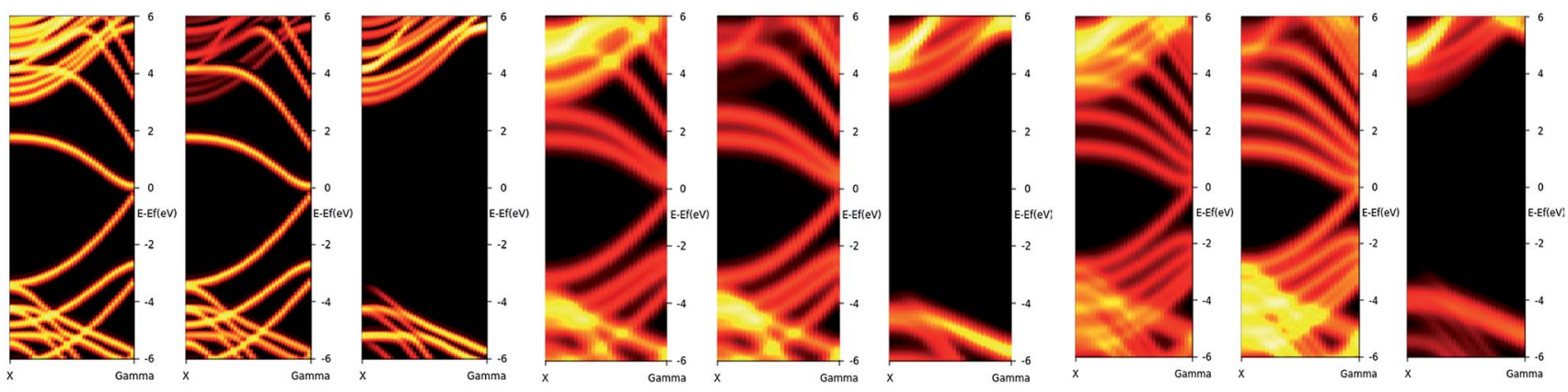

12-NZGNR

16-NZGNR

Fig. 13 Computed spin polarized electronic band structures for the three different N-doped ZGNR systems. Here, the three unites for a particular system individually represents the overall, spin-up and spin-down electrons distribution, respectively. The Fermi level is set at zero.

\section{(c) Nitrogen-doped ZGNR}

In this particular section, we will rationalize the relative changes in the electronic and magnetic properties of zigzag nanoribbons after the incorporation of nitrogen impurities in the carbon network. As we know boron doping associated with a hole formation in the network, while $\mathrm{N}$ nitrogen doping introduces an extra electron (carrier). It is worth mentioning here that a strong interaction between the edges and the impurity state in ZGNR imparts relatively strong modulation to the sublattice dependencies of the dopant properties. The stronger impact of the position of $\mathrm{N}$-impurity in the electronic and magnetic properties of the ZGNR is previously reported. ${ }^{33}$

The spin-resolved density of states (DOS) and PDOS plots for the three different $\mathrm{N}$-doped systems considered for our present study are reported in Fig. 11. The observed relatively high spin polarity in the three systems can be understood from the sharp splitting of spin up and down peaks near the Fermi level. Subsequently, the characteristics major contribution from the edge carbon atoms to the overall electronic DOS of the three systems can be interpreted from the figure. The DOS for Ndoped systems are more closely related to the undoped one. The striking difference between the $\mathrm{B}$ and $\mathrm{N}$-doped states can be recognized from the sharp splitting pattern around the Fermi level. In N-doping the splitting is almost consistent for all three systems. However, the distinct deviation in the splitting pattern with the increase in doping concentration is observed for Bdoped ZGNR. Another notable difference is the location of impurity states in the DOS spectrum. It is located above the Fermi level for nitrogen doping but for boron, the impurity states are positioned below the Fermi level.

With the simultaneous increased in the doping concentration and GNR size, the impurity states shifted more and more towards the Fermi level is also prevalent in N-doped ZGNR. This exhibits an interesting chemistry for the $\mathrm{p}$ and $\mathrm{n}$ doped impurities in the hexagonal carbon network of ZGNR. In principle, the doped $\mathrm{N}$ atom is in $\mathrm{N}^{+}$states in the network containing an extra non-bonding electron (this situation will be another way around for boron doping). The extra electron prefers to stay in the vicinity of nitrogen that introduced a donor state near the
Fermi level. ${ }^{50,51}$ The extra electron of doped N-atom should occupy spin-up state as can be seen from the PDOS plots. We know there are unpaired pi electrons along the ZGNR edges. Consequently, there should be some energetic equilibrium between the coulombic attraction of $\mathrm{N}^{+}$and the electronic correlation of the extra non-bonding electron with the pi edge electrons. At a lower level of doping concentration, the equilibrium is mainly determined by the correlation between the spin-up non-bonding electron and the spin-down pi-edge electrons and this strong correlation seems to appear the nonbonding electron at the edge atoms (near the Fermi level) and the impurity states are located far from the Fermi level. As the number of $\mathrm{N}$-atoms in the framework is increased the columbic interaction between $\mathrm{N}^{+}$and the non-bonding electrons are becoming more and more favorable than the electron correlation with edge pi-electrons and the extra non-bonding electrons try to localized in the vicinity of nitrogen atoms. This brings the impurity states closer to the Fermi level. Interestingly, the same phenomena can be observed for boron doping also but the impurity states tend to be localized below the Fermi level. This finding is quite consistent with the previous reports. ${ }^{31,47}$

Unlike boron doping, nitrogen doping induced high magnetic moment to the system. The computed magnetic moment values are 2.23, 2.00 and 2.16 BM per cell for nitrogendoped 8, 12 and 16-ZGNRs, respectively. A high degree of ferromagnetic coupling between the edge state electrons imparts a higher magnetic moment to the systems. The spin density maps for the systems are presented in Fig. 12. The spin densities are symmetrically distributed between the two edges. This is strikingly different from the B-doped case, where there is a significant reduction in spin density on of the edges. In a recent report, Jiang et al. reported a reduction in magnetization of ZGNR upon nitrogen doping. ${ }^{34}$ Our simulation also predicts the same type behavior in comparison to the undoped systems. As the edge states spin-up (or spin-down) channels of pristine ZGNR are fully occupied and spin-polarized, so the extra non-bonding electron coming from nitrogen should occupy the spin-down (or spin-up) channels and this reduces the total magnetic moment of the overall system. This can be visually interpreted from the spin density map (Fig. 12). 
Computed conduction band minima (CBM) and valence band maxima (VBM) for the three N-doped ZGNR systems are included in the ESI section (Fig. S3†). They are not showing significant variation from the B-doped one. Both CBM and VBM found to locate at the edges of ZGNR. There are no such contributions from the doping sites or carbon atoms other than the edges as we observed in case of pristine ZGNRs. The half metallic nature of the far-edge $\mathrm{N}$-doped systems can be understood from the spin-resolved band structures reported in Fig. 13. The electrons present in spin-up channels showing metallic (or semi-metallic) conductance and spin-down channels exhibit insulator behavior.

\section{Conclusions}

In conclusion, we have systematically rationalized the effect of simultaneous changes in the doping concentration and sizes on the electronic and magnetic properties of pristine, boron and nitrogen doped zigzag graphene nanoribbons. Incorporation of impurities in the far-edge doping sites, diagonally opposite to each other, induce some promising electronic and magnetic aspects in ZGNR network. All these findings can potentially influence the process of tuning the electronic and magnetic properties of ZGNR for experimental device fabrications. Eventually, increase in the size of undoped ZGNR is directly proportional to the increase in overall magnetic moment of the system whereas, boron and nitrogen doping in the crystal lattice showing the gradual decrease in the overall magnetic moment. Symmetrically substituted nitrogen and boron impurities at a far-edge (far from the edges) sites of ZGNR framework appeared to bring the impurity states more and more closer to the Fermi level. Interestingly, the presence of either boron or nitrogen atoms at the far-edge sites in the hexagonal carbon network imparts some 'half-metallic' nature to the ZGNR system as the electrons in spin-up channels are semi-metallic and spin-down electrons are insulating in nature. Our present findings suggested that the far-edge doping is also equally important as the edge doping to modulate and control the electronic properties of ZGNR. Eventually, a comparative study to understand the doping and size induced changes to the spindependent behavior of undoped to $\mathrm{p}$ and $\mathrm{n}$ doped ZGNR systems put some insights to the potential application of such systems in future spintronic devices. This is our very first attempt to predict some efficient ways to tune the electronic environment of $1 \mathrm{D}$ hexagonal carbon network for real applications based on ab initio simulation techniques.

\section{Conflicts of interest}

There are no conflicts of interest to declare.

\section{Acknowledgements}

This work was supported by research project RVO 61388963 of the Czech Academy of Sciences. We acknowledge the financial support of the Czech Science Foundation (AS, PH: P208/12/ G016). This work was supported by the Ministry of Education,
Youth and Sports from the Large Infrastructures for Research, Experimental Development and Innovations project "IT4Innovations National Supercomputing Center - LM2015070" as well as from project LO1305 (PH).

\section{References}

1 H. Petroski, The Pencil: A History of Design and Circumstance, Alfred Knopf, New York, 1989.

2 P. R. Wallace, Phys. Rev., 1947, 71, 622-634.

3 K. S. Novoselov, A. K. Geim, S. V. Morozov, D. Jiang, Y. Zhang, S. V. Dubonos, I. V. Gregorieva and A. Firsov, Science, 2004, 306, 666-669.

4 M. I. Katsnelson, K. S. Novoselov and A. K. Geim, Nat. Phys., 2006, 2, 620-625.

5 K. S. Novoselov, A. K. Geim, S. V. Morozov, D. Jiang, Y. Zhang, S. V. Dubonos, I. V. Gregorieva and A. Firsov, Nature, 2005, 438, 197-200.

6 H. Rydberg, M. Dion, N. Jacobson, F. Schröder, P. Hyldgaard, S. I. Simak, D. C. Langreth and B. I. Lundqvist, Phys. Rev. Lett., 2003, 91, 126402.

7 J. Chen, M. Vanin, Y. Hu and H. Guo, Phys. Rev. B: Condens. Matter Mater. Phys., 2012, 6, 075146.

8 T. Ozaki, K. Nishio, H. Weng and H. Kino, Phys. Rev. B: Condens. Matter Mater. Phys., 2010, 81, 075422.

9 J. Kang, F. Wu and J. Li, Appl. Phys. Lett., 2011, 98, 083109.

10 Y. Ren and K. Q. Chen, J. Appl. Phys., 2010, 107, 044514.

11 P. Kratzer, S. A. Tawfik, X. Y. Cui and C. Stampfl, RSC Adv., 2017, 7, 29112-29121.

12 M. M. Monshi, S. M. Aghaei and I. Calizo, $R S C A d v ., 2017,7$, 18900-18908.

13 X. Li, D. Zou, B. Cui, C. Fang, J. Zhao, D. Li and D. Liu, $R S C$ Adv., 2017, 7, 28124-28129.

14 P. Ruffieux, et al., Nature, 2016, 531, 489-492.

15 G. Luo, Z.-Z. Zhang, G. Wei Deng, H. Ou Li, G. Cao, M. Xiao, G. Can Guo and G. Ping Guo, Nanoscale, 2017, 9, 5608-5614.

16 G. A. Nemnes, C. Visan and A. Manolescu, J. Mater. Chem. C, 2017, 5, 4435-4441.

17 M. Guo, Y. Yang, Y. Leng, L. Wang, H. Dong, H. Liu and W. Li, J. Mater. Chem. C, 2017, 5, 4845-4851.

18 S. Kawai, et al., Science, 2016, 351, 957-961.

19 X. Li, X. Wang, L. Zhang, S. Lee and H. Dai, Science, 2008, 319, 1229-1232.

20 J. Stajic, Science, 2016, 351, 351-352.

21 T. B. Martins, R. H. Miwa, A. J. R. da Silva and A. Fazzio, Phys. Rev. Lett., 2007, 98, 196803.

22 Z. F. Wang, Q. W. Shi, Q. Li, X. Wang, J. G. Hou, H. Zheng, Y. Yao and J. Chen, Appl. Phys. Lett., 2007, 91, 53109.

23 Y. P. Chen, Y. E. Xie and J. Zhong, Phys. Lett. A, 2008, 372, 5928-5931.

24 A. N. Andriotis and M. Menon, Appl. Phys. Lett., 2008, 92, 42115.

25 T. Jayasekera and J. W. Mintmire, Int. J. Quantum Chem., 2007, 107, 3071-3076.

26 L. Pisani, J. A. Chan, B. Montanari and N. M. Harrison, Phys. Rev. B: Condens. Matter Mater. Phys., 2007, 75, 064418. 
27 E. V. Castro, N. M. R. Peres, J. M. L. B. Lopes dos Santos, A. H. Castro Neto and F. Guinea, Phys. Rev. Lett., 2008, 100, 026802.

28 M. Fujita, K. Wakabayashi, K. Nakada and K. Kusakabe, J. Phys. Soc. Jpn., 1996, 65, 1920-1923.

29 Y. Li, Z. Zhou, P. Shen and Z. Chen, ACS Nano, 2009, 3, $1952-$ 1958.

30 E. Cruz-Silva, Z. M. Barnett, B. G. Sumpter and B. V. Meunier, Phys. Rev. B: Condens. Matter Mater. Phys., 2011, 83, 155445. 31 S. S. Yu, W. T. Zheng, Q. B. Wen and Q. Jiang, Carbon, 2008, 46, 537-543.

32 F. Zou, et al., Sci. Rep., 2015, 5, 15966.

33 E. Cruz-Silva, Z. M. Barnett, B. G. Sumpter and V. Meunier, Phys. Rev. B: Condens. Matter Mater. Phys., 2011, 83, 155445.

34 J. Jiang, J. Turnbull, W. Lu, P. Boguslawski and J. Bernholc, J. Chem. Phys., 2012, 136, 014702.

35 S. Dutta and S. K. Pati, J. Mater. Chem., 2010, 20, 8207-8223. 36 H. Lee, Y. W. Son, N. Park, S. Han and J. Yu, Phys. Rev. B: Condens. Matter Mater. Phys., 2005, 72, 174431.

37 K. Wakabayshi, J. Phys. Soc. Jpn., 2002, 71, 2500-2504.

38 P. Giannozzi, et al., J. Phys.: Condens. Matter, 2009, 21, 395502.

39 J. P. Perdew, K. Burke and M. Ernzerhof, Phys. Rev. Lett., 1996, 77, 3865-3868.
40 S. Grimme, J. Comput. Chem., 2006, 27, 1787-1799.

41 H. J. Monkhorst and J. D. Pack, Phys. Rev. B: Solid State, 1975, 13, 5188-5192.

42 K. Nakada, M. Fujita, G. Dresselhaus and M. S. Dresselhaus, Phys. Rev. B: Condens. Matter Mater. Phys., 1996, 54, 1795417960.

43 L. Brey and H. A. Fertig, Phys. Rev. B: Condens. Matter Mater. Phys., 2006, 73, 235411.

44 M. Ezawa, Phys. Rev. B: Condens. Matter Mater. Phys., 2006, 73, 045432.

45 X. J. Wu and X. C. Zeng, Nano Res., 2008, 1, 40-45.

46 T. Kawai, Y. Miyamoto, O. Sugino and Y. Koga, Phys. Rev. B: Condens. Matter Mater. Phys., 2000, 62, 16349-16352.

47 T. B. Martins, A. J. R. da Silva, R. H. Miwa and A. Fazzio, Nano Lett., 2008, 8, 2293-2298.

48 R. R. Cloke, et al., J. Am. Chem. Soc., 2015, 137, 8872-8875.

49 Y. W. Son, M. L. Cohen and S. G. Louie, Nature, 2006, 444, 347-349.

50 A. H. Nevidomskyy, C. Csányi and M. C. Payne, Phys. Rev. Lett., 2003, 91, 105502.

51 J. Y. Yi and J. Bernholc, Phys. Rev. B: Condens. Matter Mater. Phys., 1993, 47, 1708-1711. 